\title{
Modeling the interactions between stimulation and physiologically induced APs in a mammalian nerve fiber: dependence on frequency and fiber diameter
}

\author{
Vijay Sadashivaiah $^{1}$ (D) $\cdot$ Pierre Sacré $^{1} \cdot$ Yun Guan $^{2,3} \cdot$ William S. Anderson $^{3} \cdot$ Sridevi V. Sarma $^{1}$
}

Received: 17 April 2018 / Revised: 18 October 2018 / Accepted: 22 October 2018

(C) Springer Science+Business Media, LLC, part of Springer Nature 2018

\begin{abstract}
Electrical stimulation of nerve fibers is used as a therapeutic tool to treat neurophysiological disorders. Despite efforts to model the effects of stimulation, its underlying mechanisms remain unclear. Current mechanistic models quantify the effects that the electrical field produces near the fiber but do not capture interactions between action potentials (APs) initiated by stimulus and APs initiated by underlying physiological activity. In this study, we aim to quantify the effects of stimulation frequency and fiber diameter on AP interactions involving collisions and loss of excitability. We constructed a mechanistic model of a myelinated nerve fiber receiving two inputs: the underlying physiological activity at the terminal end of the fiber, and an external stimulus applied to the middle of the fiber. We define conduction reliability as the percentage of physiological APs that make it to the somatic end of the nerve fiber. At low input frequencies, conduction reliability is greater than $95 \%$ and decreases with increasing frequency due to an increase in AP interactions. Conduction reliability is less sensitive to fiber diameter and only decreases slightly with increasing fiber diameter. Finally, both the number and type of AP interactions significantly vary with both input frequencies and fiber diameter. Modeling the interactions between APs initiated by stimulus and APs initiated by underlying physiological activity in a nerve fiber opens opportunities towards understanding mechanisms of electrical stimulation therapies.
\end{abstract}

Keywords Action potential interactions $\cdot$ Conduction reliability $\cdot$ Nerve fiber $\cdot$ Mechanistic model $\cdot$ Electrical stimulation

\section{Introduction}

Studying the effects of electrical stimulation on the mammalian nerve fiber has been of prime interest due to its

Action Editor: Gaute T. Einevoll

Electronic supplementary material The online version of this article (https://doi.org/10.1007/s10827-018-0703-y) contains supplementary material, which is available to authorized users.

Vijay Sadashivaiah

vjs@jhu.edu

1 Institute for Computational Medicine, Department of Biomedical Engineering, The Johns Hopkins University, Baltimore, MD 21218, USA

2 Department of Anesthesiology/Critical Care Medicine, The Johns Hopkins University School of Medicine, Baltimore, MD 21205, USA

3 Department of Neurosurgery, The Johns Hopkins University School of Medicine, Baltimore, MD 21205, USA applications in treating various diseases. Stimulation of peripheral and dorsal column fibers is used to alleviate acute and chronic pain (Shealy et al. 1967; Wall and Sweet 1967; Stidd et al. 2012; Shechter et al. 2013); stimulation of muscle fibers is used to restore sensory or motor function loss caused by nerve injury or diseases such as multiple sclerosis and cerebral palsy (Kralj and Bajd 1989; Pfurtscheller et al. 2003; Peckham and Kilgore 2013); and stimulation of the vagus nerve has been effective in treating epilepsy, depression, and anxiety (Groves and Brown 2005). In many cases, these nerve fibers have underlying physiological activity that interacts with the exogenous activity generated by the external current stimulus.

There are three main interactions that occur (Sacré et al. 2015): (i) collisions between antidromic APs from stimulation and orthodromic APs from underlying physiological activity, (ii) inter-signal loss of excitability of the fiber to an AP generated by physiological activity due to a recent AP generated by stimulus (physiological-stimulus refractory period), and vice versa (stimulus-physiological refractory period), and (iii) intra-signal loss of excitability 
of the fiber to an AP generated by stimulus due to a recent stimulus AP (stimulus-stimulus refractory period) and to an AP generated by physiological activity due to a recent AP generated by physiological activity (physiological-physiological refractory period). In order to better understand and optimally design stimulation therapies for diseases, it is necessary to understand when and how each of these interactions occur under different stimulation protocols.

A few experimental studies have investigated and/or exploited interactions between APs generated by stimulus and underlying physiological activity. Collision block was used by Iggo in cats as a technique to isolate a single unmyelinated nerve fiber from vagal afferent fibers (Iggo 1958). Iggo stimulated the fiber at both ends and studied collisions between antidromic activation at one end of the fiber with orthodromic activation from the other end. Collision block was also exploited to artificially block unwanted physiological activity in the sciatic nerve (van den Honert and Mortimer 1981). A periodic train of unidirectional antidromic APs was used to block underlying orthodromic activity. These studies considered only collisions and were limited to deterministic inputs (single pulse or periodic train of impulses).

There have also been computational efforts to study the effects of stimulation on a mammalian nerve fiber. In particular, biophysical conductance-based models of nerve fibers have been developed to study their excitability properties (McIntyre et al., 1989; Reilly, 1999; Wesselink et al., 2002, to name a few). These studies characterize the effects of stimulation on the fiber, such as activation threshold and conduction velocity, by modeling different nerve geometries (single cable or double cable), different extracellular medium compositions, and different electrode configurations, but do not study interactions between APs generated by stimulus with underlying physiological activity in the fiber.

A more recent study developed a simple computational model based on the speed of conduction and refractory periods to capture these interactions (Crago and Makowski 2014). Two inputs were applied to different sites along the fiber: (i) the physiological activity was modeled as a spike train whose mean firing rate is generated by a normal distribution and (ii) the external stimulus was modeled as a periodic train of APs. Collision block and inter-signal loss of excitability were studied as a function of the mean firing rate of APs generated by physiological activity. To our knowledge, this functional model is the first to investigate interactions rigorously but cannot identify nonlinear effects like relative refractory periods that may arise in the biophysical fiber model. Furthermore, underlying physiological activity may not be well characterized by a normally distributed firing pattern (Jänig et al. 2009). Variable patterns (bursts, Poisson, etc.) of stimulus are more realistic characterizations of physiological response processes (Bruns et al. 2009). In our recent study (Sadashivaiah et al. in press(a)), we constructed a similar functional model and compared the conduction properties of the fiber to a high dimensional mechanistic model. In this study, we aimed to further investigate the interactions between APs generated by stimulus with underlying physiological activity in the fiber using a mechanistic model.

Specifically, we constructed a mechanistic model of an unbranched, myelinated nerve fiber based on biophysical principles. This fiber receives two inputs: the underlying physiological activity initiated at the terminal end of the fiber and the external stimulus applied to the middle of the fiber (see Fig. 1). We observed and characterized all three types of interactions between antidromic APs generated by a deterministic periodic external stimulus and orthodromic physiological APs generated by a stochastic Poisson process. We computed conduction reliability, defined as the ratio of the number of physiological APs that make it to the somatic end of the fiber over the total number of physiological APs entering nerve fiber. We analyzed conduction reliability using a mechanistic model of relay neurons in the thalamus in a previous study (Agarwal and Sarma 2012).

We varied the frequency of the input signals and computed conduction reliability for different fiber diameters. Our results demonstrate that the conduction reliability strongly depends on both physiological and stimulus input frequencies and weakly depends on the diameter of the

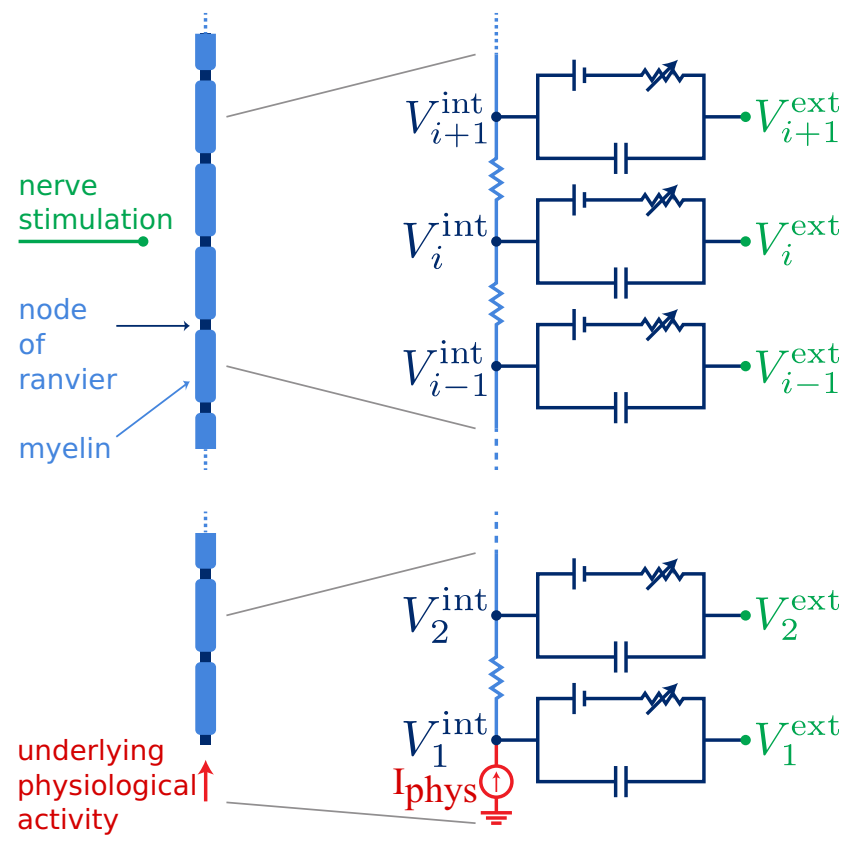

Fig. 1 Mechanistic model of nerve fiber. Our model of extracellular electrical stimulation of myelinated nerve fibers includes the underlying physiological activity as a current source, $I_{\text {phys }}$ at terminal end of the nerve fiber 
nerve fiber. For low input frequencies, more than $95 \%$ of physiological APs reach the somatic end of the nerve fiber. As input frequencies increase, conduction reliability decreases. We show that the fraction of APs that make it to the somatic end of the fiber due to stimulus and physiological sources are uniformly distributed when the ratio of their frequencies is unity. The number and type of interactions vary highly with both input frequencies and fiber diameter. Physiological - stimulus loss of excitability occurs most frequently and collisions occur least frequently.

This study demonstrates that complex interactions occur between physiological and stimulus-induced APs along a nerve fiber and that it is useful to characterize these interactions to better understand the mechanisms of action of electrical stimulation used to treat diseases of the nervous system.

\section{Methods}

\subsection{Mechanistic model of a nerve fiber}

Methods used in this study are derived from (Sadashivaiah et al. 2017). Here, we describe our model of a myelinated nerve fiber that incorporates extracellular electrical stimulation and underlying physiological activity (see Fig. 1). This axonal model is relatively simple in comparison to other higher order approximations of mammalian nerve fibers (McIntyre et al. 2002), as our main interest is to study the effect of interactions between APs generated by stimulation with underlying activity.

\subsubsection{Myelinated nerve fiber}

A myelinated nerve fiber is a cylindrical active membrane (axon), tightly wrapped in an insulating myelin sheath. This myelin sheath is interrupted periodically, leaving short gaps where the axonal membrane is exposed. Following McNeal's model (McNeal 1976), we represent a myelinated nerve fiber by a series of compartment elements linked by intracellular conductances. The dynamics of the membrane potential $V_{i}=V_{i}^{\text {int }}-V_{i}^{\text {ext }}$ at node $i$ (where $V_{i}^{\text {int }}$ and $V_{i}^{\text {ext }}$ are the intracellular and extracellular potentials) read as follows

$$
\begin{aligned}
C_{\mathrm{m}} \dot{V}_{i}+\sum_{k \in \mathcal{K}} I_{i, k}= & g_{\mathrm{a}}\left(V_{i-1}-2 V_{i}+V_{i+1}\right) \\
& +g_{\mathrm{a}}\left(V_{i-1}^{\mathrm{ext}}-2 V_{i}^{\mathrm{ext}}+V_{i+1}^{\mathrm{ext}}\right),
\end{aligned}
$$

where $C_{\mathrm{m}}$ is the membrane capacitance and $g_{\mathrm{a}}$ is the internodal conductance. Ionic currents $I_{i, k}$ at node $i$ include a sodium, a fast potassium, and a slow potassium ion channel, as well as a leakage current across the membrane based on the Frankenhaeuser-Huxley model (Frankenhaeuser and
Huxley 1964), adjusted to experimental data of human sensory fibers at $37^{\circ} \mathrm{C}$ (Schwarz et al. 1995; Wesselink et al. 1999). A complete description of the fiber model and its parameters is presented in the Appendix.

\subsubsection{Electrical field potential generated by stimulation}

The extracellular medium surrounding a nerve fiber is composed of different regions (epidural fat, cerebrospinal fluid, white matter, grey matter), which have different conduction properties (Struijk et al. 1991). In addition, the electrode can also take various shapes (single contact or array of contact) and various configurations (monopolar, bipolar, or other) (Medtronic Neuromodulation 2007). We assume that the extracellular medium is infinite, isotropic and ohmic with the electrode represented by point sources at the center $\vec{x}_{j}^{\mathrm{c}}$ of each contact. Therefore, the electrical potential field at time $t$ and position $\vec{x}$ is given by

$\varphi(t, x)=\sum_{j \in \mathcal{C}} \frac{1}{4 \pi \sigma_{\mathrm{m}}\left\|\vec{x}-\vec{x}_{j}^{\mathrm{c}}\right\|_{2}} I_{j}^{\mathrm{stim}}(t)$,

where $I_{j}^{\text {stim }}$ is the current of point source $j$ and $\sigma_{\mathrm{m}}$ is the extracellular medium conductivity. The extracellular potential at node $i$ is given by $V_{i}^{\text {ext }}(t)=\varphi\left(t, \vec{x}_{i}\right)$, where $\vec{x}_{i}$ is the position of node $i$.

\subsubsection{Underlying physiological activity}

The underlying physiological activity in fibers spans a broad frequency range and exhibits various patterns (Kajander and Bennett 1992): regular spike discharge, regular discharge of doublet spikes, bursting patterns, sporadic activity with no regular or predictable firing pattern, etc.

We represent underlying activity generated at the terminal end of the nerve fiber by replacing a 'sealedend' boundary condition by a current source. Therefore, the dynamics of the first node (from bottom) becomes

$$
\begin{aligned}
C_{\mathrm{m}} \dot{V}_{1}+\sum_{k \in \mathcal{K}} I_{1, k}= & g_{\mathrm{a}}\left(V_{2}-V_{1}\right) \\
& +g_{\mathrm{a}}\left(V_{2}^{\text {ext }}-V_{1}^{\text {ext }}\right)+I^{\text {phys }}(t),
\end{aligned}
$$

where the input $I^{\text {phys }}(t)$ represents the activation current.

\subsection{Stimulation protocols}

The stimulation current $\left(I^{\operatorname{stim}}(t)\right)$ had a constant frequency $\left(f^{\text {stim }}\right)$ of symmetrical biphasic pulses with an amplitude ranging from $1.8-2.5 \mathrm{~mA}$ (that is, $150 \%$ of the activation threshold, see Table 1) a duration of $350 \mu$ s (Mortimer and Bhadra 2004). We consider stimulation frequencies ranging from $1-51 \mathrm{~Hz}$ with a step size of $4 \mathrm{~Hz}$ (Simulations in 
Table 1 Amplitude of physiological and stimulus inputs for mechanistic model simulations

\begin{tabular}{lccr}
\hline & \multicolumn{3}{l}{ Fiber diameter $(\mu \mathrm{m})$} \\
\cline { 2 - 4 } & 6 & 9 & 12 \\
\hline Physiological input amplitude (nA) & 5.0 & 6.0 & 7.0 \\
Stimulus input amplitude (mA) & -2.5 & -2.0 & -1.8 \\
\hline
\end{tabular}

supplementary section have stimulation frequencies ranging $1-200 \mathrm{~Hz}$ with a step size of $10 \mathrm{~Hz}$ ).

As a first step, we modeled the underlying physiological activity $\left(I^{\text {phys }}(t)\right)$ as a Poisson train of square pulses with an amplitude of 5-7 nA (that is, $150 \%$ of the activation threshold) and a duration of $1 \mathrm{~ms}$. Therefore, the instantaneous firing rate $\left(\lambda^{\text {phys }}\right)$ is assumed constant, ranging from $1-51 \mathrm{~s}^{-1}$ with a step size of $4 \mathrm{~s}^{-1}$, a typical range for motor and sensory firing activity (Katz 1950; De Luca et al. 1982). (Simulations in supplementary section have instantaneous firing rate $\lambda^{\text {phys }}$ ranging $1-200 \mathrm{~s}^{-1}$ with a step size of $10 \mathrm{~s}^{-1}$ ).

Several studies have successfully used random process models to generate the statistics of spike trains. Troy and Robson (1992) found that the interval statistics of spike trains recorded from retinal ganglion cells was well fit by gamma-distributed intervals. Similarly, the inter-spike interval distributions computed from visual area (V5/middle temporal) was well fit by a Poisson process (Bair et al. 1994).

\subsection{Interactions between stimulation induced and underlying physiological activity}

We identified different types of interactions occurring between simulation-evoked activity and underlying physiological activity. In Fig. 2, each panel represents a typical line plot for each interaction type. In these panels, a dot is an AP at node position $\vec{z}$ along the fiber and at time $t$ (see zoomedin section of panel 1). The stimulation input triggers the orthodromic and antidromic propagations of an AP wave (green dots) from the stimulation position at the fiber center toward the end points. The physiological input triggers the orthodromic propagation of an AP wave (red dots) from terminal end to the somatic end of the fiber. Gray dots indicate the fiber response of each input in the absence of the other, that is, artificially without interaction (e.g., if no collision occurs and the APs just cross each other). The interaction type depends on the timing of both inputs generating these activities.

1. A collision occurs when the orthodromic physiological AP wave and the antidromic stimulation-evoked AP wave meet and cancel each other. This happens if a physiological pulse is triggered slightly before or after a stimulation pulse, that is, $t_{j}^{\text {phys }} \in\left[t_{i}^{\mathrm{stim}}-t^{\mathrm{ic}}, t_{i}^{\mathrm{stim}}+t^{\mathrm{ic}}\right)$.

2. A phys-stim loss of excitability occurs when the stimulation input doesn't excite the nerve fiber due to the recent passage of the orthodromic physiological AP wave. This happens if a physiological pulse is triggered before a stimulation pulse, that is, $t_{j}^{\text {phys }} \in\left[t_{i}^{\text {stim }}-t^{\text {ic }}-\right.$ $\left.\Delta t^{\mathrm{I}}, t_{i}^{\mathrm{stim}}-t^{\mathrm{ic}}\right)$.

3. A stim-phys loss of excitability occurs when the physiological input doesn't excite the nerve fiber due to the recent passage of the antidromic stimulationevoked AP wave. This happens if a physiological pulse is triggered after a stimulation pulse, that is, $t_{j}^{\text {phys }} \in$ $\left[t_{i}^{\text {stim }}+t^{\mathrm{ic}}, t_{i}^{\mathrm{stim}}+t^{\mathrm{ic}}+\Delta t^{\mathrm{II}}\right)$.

Here, $t^{\text {ic }}$ is the conduction time between the physiological AP generator and the stimulus location, $\Delta t^{\mathrm{I}}$ is the

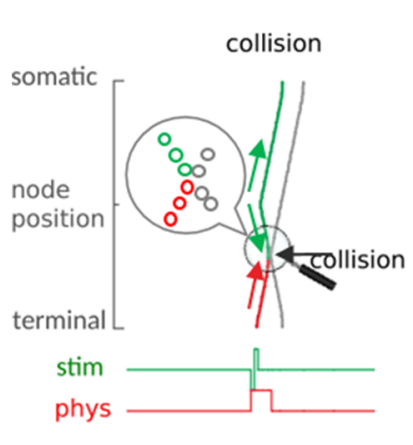

loss of excitability physiological - stimulation stimulus - physiological
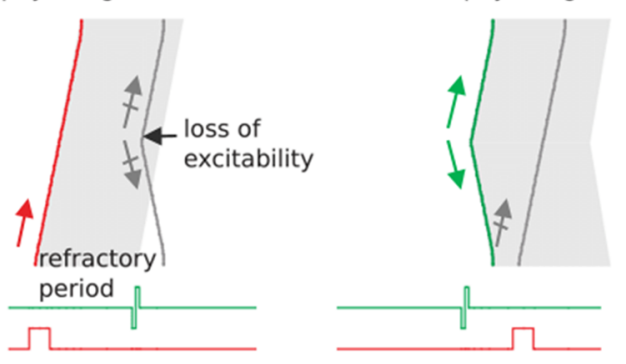

loss of excitability stimulus - stimulus

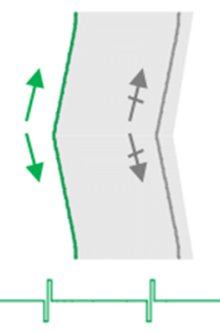

loss of excitability physiological - physiological

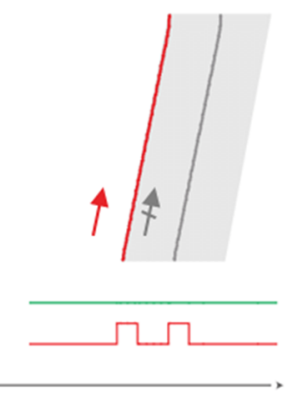

Fig. 2 Different interactions between stimulation-evoked activity and physiological activity are illustrated by their line plots (horizontal axis is time, vertical axis is position along the nerve fiber). Each dot corresponds to an AP at a given time $t$ and position $\vec{z}$ along the fiber. Red and green dots indicate the fiber response to physiological and stimulation inputs, respectively. Physiological AP waves travel orthodromically from terminal end to the somatic end of the fiber; time

stimulation-evoked AP waves travel orthodromically and antidromically from the center to the extremities of the fiber. Gray dots indicate the fiber response in the absence of interactions (collision or loss of excitability), that is, the AP wave that would be produced by the corresponding input if it was not perturbed by the activity induced by another input 
physiological-stimulus refractory period and $\Delta t^{\mathrm{II}}$ is the stimulus-physiological refractory period. In addition to these interactions, we also identify 'self-interactions' or 'intra-signal' loss of excitability, that is, interactions between activities generated by the same input.

1. A stim-stim loss of excitability occurs when the stimulation input doesn't excite the nerve fiber due to the recent stimulation of the fiber. This happens if the frequency of stimulation is too high, that is, $t_{j+1}^{\text {stim }} \in\left[t_{j}^{\text {stim }}+\right.$ $\left.\Delta t^{\mathrm{III}}\right)$.

2. A phys-phys loss of excitability occurs when a physiological input doesn't excite the nerve fiber due to a recent physiological input. This happens if two consecutive Poisson pulses are initiated too close to each other, that is, $t_{j+1}^{\text {phys }} \in\left[t_{j}^{\text {phys }}+\Delta t^{\mathrm{IV}}\right)$.

Here, $\Delta t^{\mathrm{III}}$ is the stimulus - stimulus refractory period and $\Delta t^{\mathrm{IV}}$ is the physiological-physiological refractory period.

\subsection{Conduction reliability}

When stimulation is applied to a nerve fiber, it ultimately is interfering with the underlying physiological activity that travels from one end of the fiber to the other. To succinctly quantify the effects of stimulation on the fiber activity, we wanted to capture how the stimulation influences the physiologically generated APs that make it to the somatic end of the fiber. To quantify the effects of stimulation on the nerve fiber, we define the following conduction reliability metric:

$R_{\text {phys }}\left(\lambda^{\text {phys }}, f^{\text {stim }}\right)=\frac{\# \text { of conducted phys APs }}{\text { total \# of phys APs }}$,

where conducted APs are underlying physiological inputs that travel from somatic end to the terminal end of the fiber. This metric captures the effect that the stimulation has on the underlying physiological activity. If $R_{\text {phys }}=$ 1 , then the stimulation has no effect; and if $R_{\text {phys }}=0$, then the stimulation blocks all physiological activity from transmitting to the brain.
We may also be interested in understanding the stimulation induced orthodromic APs that make it to the somatic end of the fiber $\left(R_{\text {stim }}\right)$,

$R_{\text {stim }}\left(\lambda^{\text {phys }}, f^{\text {stim }}\right)=\frac{\# \text { of conducted stim APs }}{\text { total \# of stim APs }}$.

Finally, we also look at the overall conduction of APs,

$R_{\text {all }}\left(\lambda^{\text {phys }}, f^{\text {stim }}\right)=\frac{\# \text { of conducted (phys }+ \text { stim) APs }}{\text { total \# of (phys }+ \text { stim) APs }}$.

We keep track of APs in both space and time as shown in Fig. 2, which was generated via simulations. Specifically, consider the leftmost panel in Fig. 2, which denotes a collision interaction. The red line is discretized (as shown in the zoomed-in panel), where each point represents the position of the AP generated by the physiological input at the terminal end of the fiber. The green line is also discretized and represents the APs (orthodromic and antidromic) generated by stimulation. In this case, the two inputs occur at the same time, thus right after they are applied there are 3 APs on the fiber. The physiological AP is traveling up the fiber, until it collides with the antidromic AP traveling down the fiber. The grey line is the trajectory that the physiological AP would have taken if there was no collision. However, after the time point when the collision occurs, the only action potential that exists is close to the somatic end of the fiber, which is the orthodromic AP from the stimulation input. Based on the spatiotemporal shape of the AP trajectory along the fiber, transmitted APs were classified as either of stimulus or physiological origin.

\subsection{Simulation protocols}

For this study, we consider a monopolar electrode placed $3.5 \mathrm{~mm}$ from the center of a $10 \mathrm{~cm}$-long nerve fiber. We consider three different diameters of nerve fibers in our simulations $(6,9$ and $12 \mu \mathrm{m})$. Model parameters are listed for each diameter in Table 2. These values are derived by stimulating the mechanistic model with one physiological and one stimulus AP. Results presented in the next section are drawn from 50 simulations for each
Table 2 High-dimensional mechanistic model parameters for a $10 \mathrm{~cm}$ long myelinated nerve fiber

\begin{tabular}{llll}
\hline & \multicolumn{2}{l}{ Fiber diameter $(\mu \mathrm{m})$} & \\
\cline { 2 - 4 } & 6 & 9 & 12 \\
\hline speed of AP conduction $\left(\mathrm{m} \mathrm{s}^{-1}\right)$ & 41.66 & 66.67 & 90.91 \\
physiological-stimulus refractory period $-\Delta t^{\mathrm{I}}(\mathrm{ms})$ & 9.5 & 7.8 & 7.7 \\
stimulus-physiological refractory period $-\Delta t^{\mathrm{II}}(\mathrm{ms})$ & 4.3 & 3.9 & 4.3 \\
stimulus-stimulus refractory period $-\Delta t^{\mathrm{III}}(\mathrm{ms})$ & 8.5 & 7.0 & 6.2 \\
physiological-physiological refractory period $-\Delta t^{\mathrm{IV}}(\mathrm{ms})$ & 3.2 & 3.5 & 4.0 \\
\hline
\end{tabular}


[physiological rate, stimulus frequency] pair. These results are generated by running Monte Carlo simulations, that is, running the same simulation multiple times for different realizations of the stochastic input and computing statistics (mean and standard deviation) of conduction reliability. All the mechanistic model simulations were performed in the NEURON simulation environment (Hines and Carnevale 1997). Analysis of data was performed using MATLAB, MathWorks. Individuals interested in reproducing the results of this study or using these models in their own work can find the source-files on github (Sadashivaiah 2018).

\section{Results}

\subsection{Conduction reliability}

First, we simulated a range of stimulus frequencies $\left(f^{\text {stim }}\right.$ $=1-51 \mathrm{~Hz})$ and physiological rates $\left(\lambda^{\text {phys }}=1-51 \mathrm{~s}^{-1}\right)$ and computed conduction reliability. Results are displayed in the form of "conduction maps".

\subsubsection{Conduction map of all APs}

APs, irrespective of the source (stimulus vs physiological), can have similar firing patterns at the supra-spinal centers. In order to quantify this, we plot the overall conduction, that is, the ratio of APs reaching the somatic end of the nerve fiber over the APs entering the terminal end of the nerve fiber (see Fig. 3).

Conduction maps indicate that the reliability value depends on both the frequency of stimulation and rate of physiological activity, and also varies with the fiber diameter. At low physiological rates $\left(\lambda^{\text {phys }}<10 \mathrm{~Hz}\right)$, reliability is high $\left(R_{\text {all }}>0.9\right)$ and doesn't change with respect to the stimulus frequency. As the physiological rate increases, reliability value decreases. Increasing the fiber diameter increases the reliability at each frequency-rate pair $\left(f^{\text {stim }}, \lambda^{\text {phys }}\right)$. The standard deviation of conduction reliability is small $(0.01$ - 0.02) and doesn't seem to depend on either stimulus frequency, physiological rate or fiber diameter. To study the influence of stimulus on underlying physiological activity, we consider the conduction of physiological APs and stimulus generated APs separately.

\subsubsection{Conduction map of underlying physiological APs}

Underlying physiological activity interacts with the stimulus APs before reaching the somatic end of nerve fiber. Due to it's stochastic nature, the overall conduction is modulated by underlying physiological activity. An AP generated by the physiological source may collide with an antidromic stimulus AP, or fail to elicit and AP on the fiber due to stimulus-physiological loss of excitability, or fail to elicit an AP on the fiber due to physiological-physiological loss of excitability. The conduction reliability map for physiological APs, $R_{\text {phys }}$ is shown in Fig. 4.

For a low stimulus frequency and low physiological rate $\left(f^{\text {stim }}<10 \mathrm{~Hz}, \lambda^{\text {phys }}<10 \mathrm{~s}^{-1}\right)$, reliability values are close to $1(100 \%)$. Increasing either the stimulus frequency or the physiological rate, reduces reliability. Increasing the fiber diameter decreases reliability at each frequency pairs. Specifically, the relay of physiological APs reduces by an order of $15 \%$ at higher fiber diameters (see Fig. 4c). The standard deviation of physiological conduction reliability increases with increasing $\lambda^{\text {phys }}$.
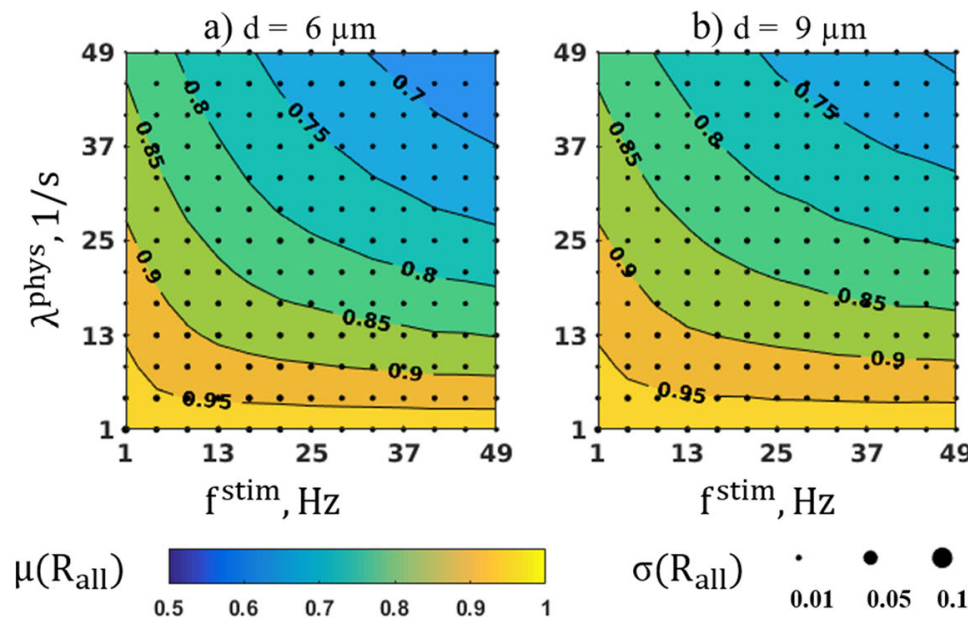

0.01

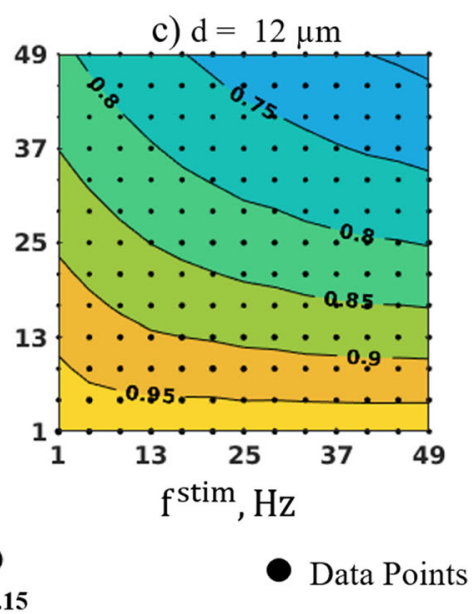

frequency (X-axis). Black dots represent the data points. Color gradient represents the mean of reliability values $\left(\mu\left(R_{\text {all }}\right)=[0.5-1]\right)$. Size of the dot represents the standard deviation $\left(\sigma\left(R_{\text {all }}\right)\right)$ of reliability values 


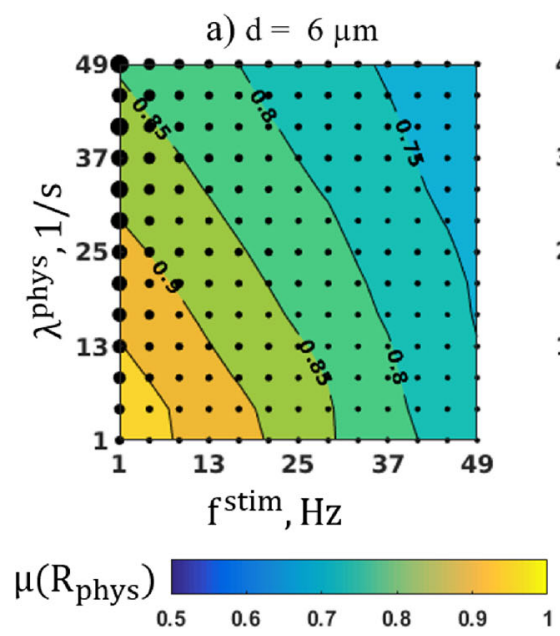

Fig. 4 Conduction maps of physiological APs for 6, 9 and $12 \mu \mathrm{m}$ diameters (d). Contour representation of reliability values for a range $\left(\lambda^{\text {phys }}=1-51 \mathrm{~s}^{-1}\right)$ of physiological rate $($ Y-axis $)$ and $\left(f^{\text {stim }}=1-51\right.$ $\mathrm{Hz}$ ) stimulus frequency (X-axis). Black dots represent the data points.

In order to quantify the effects of high frequency stimulus and high physiological rate on conduction, we ran a simulation for $f^{\text {stim }}=1-200 \mathrm{~Hz}, \lambda^{\text {phys }}=1-200 \mathrm{~s}^{-1}$ (see supplementary Fig. S1). We observe a band of stimulation frequencies spanning $5-10 \mathrm{~Hz}$, where conduction reliability approaches zero (for example, in Fig. S1(c), this band is around $150 \mathrm{~Hz}$ ).

\subsubsection{Conduction map of orthodromic stimulus APs}

A current pulse from an external stimulus can elicit two APs, one traveling orthodromically and the other antidromically. Although APs from physiological source interact with antidromic stimulus APs, it is important to characterize orthodromic stimulus APs. These are the APs that reach the somatic end of the nerve fiber in addition to physiological APs. An orthodromic AP from the stimulus source elicits a refractory period on the nerve fiber causing a stimulusphysiological loss of excitability or a stimulus-stimulus loss of excitability. We compute conduction reliability for APs induced by the stimulus, $R_{\text {stim }}$ and illustrate the conduction map in Fig. 5.

We see that conduction reliability values vary linearly with respect to $\lambda^{\text {phys }}$. For low physiological frequencies $\left(\lambda^{\text {phys }}<10 \mathrm{~Hz}\right)$, reliability values are close to $1(100 \%)$. Increasing the physiological rate reduces reliability. Reliability of stimulus APs is not significantly affected by stimulus frequency, however, increasing the fiber diameter increases reliability. Specifically, the relay of stimulus APs increases by an order of $15 \%$ for larger fiber diameters (see Fig. 5c). The standard deviation of stimulus conduction reliability increases with increasing $f^{\text {stim }}$. b) $\mathrm{d}=9 \mu \mathrm{m}$

b......
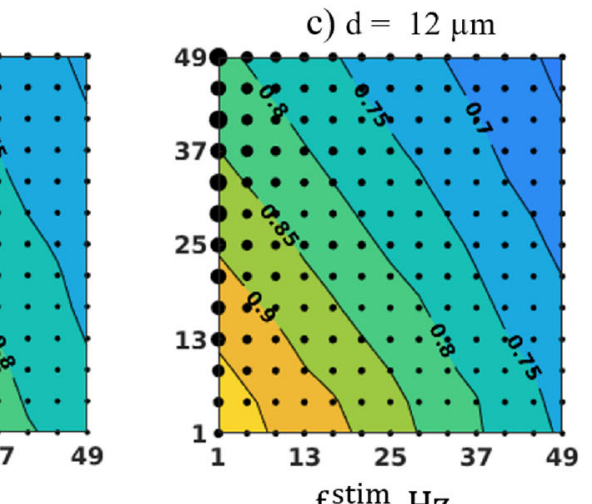

$\mathrm{f}^{\text {stim }}, \mathrm{Hz}$

- Data Points
Color gradient represents the mean of reliability values $\left(\mu\left(R_{\text {phys }}\right)=\right.$ $[0.5-1])$. Size of the dot represents the standard deviation $\left(\sigma\left(R_{\text {phys }}\right)\right)$ of reliability values

\subsection{Interaction statistics}

The mechanisms of interactions between APs generated by stimulus and physiological inputs can be disparate (see Fig. 2). They depend mainly on the frequency of both the stimulus and physiological sources, biophysical properties of the nerve fiber, and the geometry of nerve fiber. Conduction maps give a basic representation of our reliability metric but do not quantify the various interactions occurring in the nerve fiber. To address this, we consider 5 different regions on the conduction map and examine the interactions statistics in each of these locations. Specifically, we consider the

1. low stimulus frequency $(5 \mathrm{~Hz})$ and low physiological rate $\left(5 \mathrm{~s}^{-1}\right)$,

2. low stimulus frequency $(5 \mathrm{~Hz})$ and high physiological rate $\left(45 \mathrm{~s}^{-1}\right)$,

3. medium stimulus frequency $(25 \mathrm{~Hz})$ and medium physiological rate $\left(25 \mathrm{~s}^{-1}\right)$,

4. high stimulus frequency $(45 \mathrm{~Hz})$ and low physiological rate $\left(5 \mathrm{~s}^{-1}\right)$, and

5. high stimulus frequency $(45 \mathrm{~Hz})$ and high physiological rate $\left(45 \mathrm{~s}^{-1}\right)$ regions represented in Fig. 6a.

We also compute the fraction of APs reaching the somatic end of nerve fiber due to the stimulus versus the physiological source. In order to test the difference between means of interaction counts in 6,9 and $12 \mu \mathrm{m}$ diameter fibers, we conducted a 3 group one-way ANOVA. Statistical significance is presented using asterisk $(*)$ in Fig. 6c-f. p values corresponding to these asterisks are, $(*) p<0.05$, 

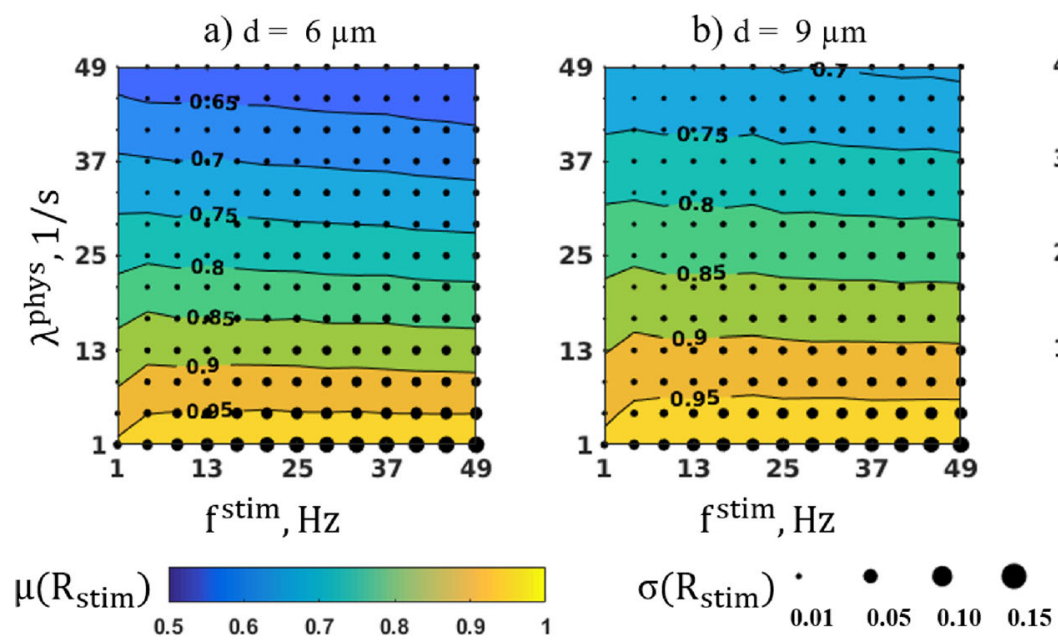

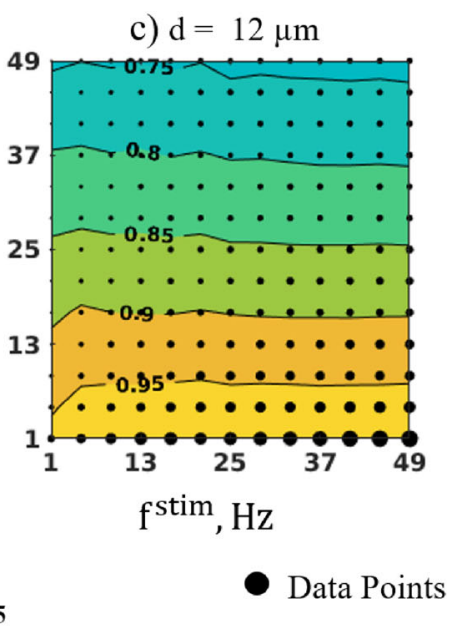

Fig. 5 Conduction maps of stimulus APs for 6, 9 and $12 \mu$ ms diameters $(\mathbf{d})$. Contour representation of reliability values for a range ( $\lambda^{\text {phys }}$ $\left.=1-51 \mathrm{~s}^{-1}\right)$ of physiological rate $(\mathrm{Y}$-axis $)$ and $\left(f^{\text {stim }}=1-51 \mathrm{~Hz}\right)$ stimulus frequency (X-axis). Black dots represent the data points. Color

(**) $p<1 E^{-2},(* * *) p<1 E^{-3}$. Further details including $F$ scores and $p$ values are listed in Supplementary Materials (see Table S1).

We summarize our observations next.

1. When the physiological rate and stimulus frequency are equal, that is, $f^{\text {stim }} / \lambda^{\text {phys }}=1$ (regions $1,3 \& 5$ ), the fraction of endpoint APs is constant and relatively close to 0.5 . Increasing the physiological rate (or stimulus frequency), that is, $f^{\text {stim }} / \lambda^{\text {phys }}<1$, region 2 (or $f^{\text {stim }} / \lambda^{\text {phys }}>1$, region 4 ) increases the fraction of somatic APs due to physiological (or stimulus) source. Increasing the fiber diameter increases the fraction of somatic APs due to stimulus.

2. Collisions occur less frequently when compared to all other types of interactions (see Fig. 6). For regions 1, 3 $\& 5$, the number of collisions increases gradually with respect to $f^{\text {stim }}$ and $\lambda^{\text {phys }}$. Both regions 2 and 4 , have similar collision counts. Interestingly, the number of collisions increase for the $9 \mu \mathrm{m}$ fiber but then decreases for the $12 \mu \mathrm{m}$ fiber.

3. Physiological-physiological loss of excitability interaction increases with respect to $f^{\text {stim }}$ and $\lambda^{\text {phys }}$ for regions $1,3 \& 5$. Both regions 2 and 5 have high physiologicalphysiological loss of excitability relative to the other regions and increasing the fiber diameter increases the number of these interactions.

4. Physiological-stimulus loss of excitability occurs most frequently. For regions $1,3 \& 5$, the number of interactions significantly increases with respect to $f^{\text {stim }}$ and $\lambda^{\text {phys }}$. Both regions 2 and 4 have low physiologicalstimulus loss of excitability relative to the other regions, and increasing the fiber diameter decreases the number of these interactions. gradient represents the mean of reliability values $\left(\mu\left(R_{\text {stim }}\right)=[0.5-\right.$ $1])$. Size of the dot represents the standard deviation $\left(\sigma\left(R_{\text {stim }}\right)\right)$ of reliability values

5. Stimulus-physiological loss of excitability significantly increases for regions $1,3 \& 5$. Regions 2 and 4 have low stimulus-physiological loss of excitability relative to the other regions, and increasing the diameter of fiber increases the number of interactions, but only slightly.

To get a sense of interaction distributions across map regions, we identify the dominant interaction at any given point on the reliability maps for 6,9 and $12 \mu \mathrm{m}$ diameters (see Fig. S3). We see that the physiological - stimulus loss of excitability is the dominating interaction type, covering more than $50 \%$ of the map. Collisions occur the least at each [frequency, rate] pair, and increasing the diameter increases the region which is dominated by physiologicalphysiological loss of excitability.

\section{Discussion}

In this study, we investigated the interactions between APs traveling along a mammalian nerve fiber induced by both electrical stimulation and physiological activity. To our knowledge, this is the first mechanistic modeling effort to quantify these interactions. We found that the conduction reliability of APs depends on the frequency of stimulation and the rate of the physiological activity. Specifically, overall conduction reliability $R_{\text {all }}$ is modulated by the rate of physiological activity. This is also supported by the dominant occurrence of physiological-stimulus loss of excitability interactions. Conduction reliability of physiological APs, $R_{\text {phys }}$ is high for low stimulus frequencies and low physiological rates and decreases with an increase of either stimulus frequency or physiological rate. On the other hand, conduction reliability of stimulus APs, $R_{\text {stim }}$ is 

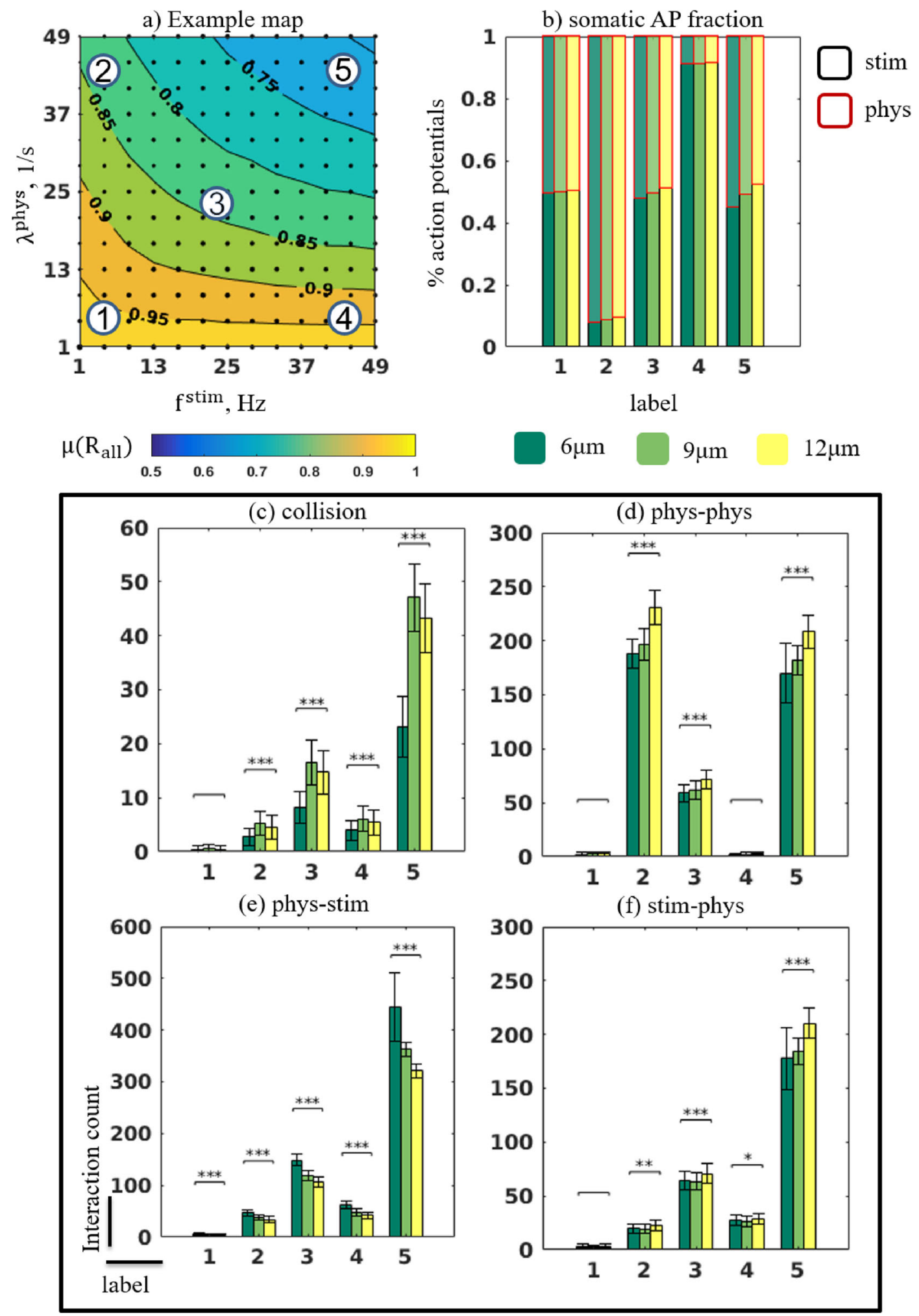

Fig. 6 Interaction statistics at various regions of conduction map for 6,9 and $12 \mu \mathrm{m}$ diameters. a. An example conduction map representing regions of interest (ROI) labelled 1 through 5. b. Fraction of somatic APs due to stimulus and physiological sources for labels 1-5. Lower region represents the fraction due to stimulus (black outline) and the higher represents the fraction due to physiological (red outline). Each group of 3 bars represent the fractions for 6,9 and $12 \mu \mathrm{m}$ diameters. c-f.
Interaction count at labels 1-5 for collision, physiological-physiological loss of excitability, physiological-stimulus loss of excitability and stimulus-physiological loss of excitability respectively. Each bar in a group (of 3) represents the count for $6 \mu \mathrm{m}$ (dark green), $9 \mu \mathrm{m}$ (green), $12 \mu \mathrm{m}$ (yellow) diameters. Significance testing was performed at each ROI for 6, 9 and $12 \mu \mathrm{m}$ diameters using one-way ANOVA (* $\left.p<0.05, * * p<1 E^{-2}, * * * p<1 E^{-3}\right)$ 
only modulated by physiological rate. Increasing the fiber diameter decreases $R_{\text {phys }}$ but increases $R_{\text {stim }}$ at all [stimulus frequency, physiological rate] pairs. The fraction of somatic APs from the stimulus and physiological sources remain constant when $f^{\text {stim }}=\lambda^{\text {phys }}$, but the individual interaction counts vary. Overall, collisions occur least frequently and physiological-stimulus losses of excitability occur most frequently.

\subsection{Conduction reliability of APs}

Various modeling studies use different metrics to capture the effects of electrical stimulation on a mammalian nerve fiber, but the most prominent measure studied is output firing rate (the number of APs at somatic end of nerve fiber per unit time). In our study, we use a novel metric, conduction reliability, which captures the ratio of the number of APs reaching the somatic end of a fiber over the total number of APs generated in the nerve fiber. This essentially captures loss of information on the fiber due to AP interactions. Our metric is particularly useful in that it isolates the effect of stimulation on underlying physiological activity.

As expected, we found that the conduction reliability is high for low physiological rates and/or stimulus frequencies, because fewer interactions occur between activity evoked by simulation and underlying physiological activity. As the frequency of the stimulus and/or rate of the physiological input increases, conduction reliability decreases (see Figs. 3, 4 and 5). Specifically, the conduction of physiological APs is highly regulated by both stimulus frequency and physiological rate. In our model, for a given nerve fiber of length $l$, a physiological AP travels twice the length (l) compared to a stimulus AP $(l / 2)$ to reach the somatic end of the nerve fiber. Hence the former is susceptible to more interactions. Conduction reliability of stimulus APs is independent of stimulus frequency since the only interaction influencing this value are physiological-stimulus loss of excitability interactions (which depends on the physiological rate).

Increasing the fiber diameter essentially translates to faster conduction speed (Hursh 1939). Therefore bigger fibers require higher stimulus frequencies to achieve the relay properties observed for lower stimulus frequencies in smaller fibers. As the conduction speed of APs increase, the time window for interactions decreases. This explains the increase in overall conduction reliability at each [stimulus frequency, physiological rate] pair for larger diameter fibers (see Fig. 3). We see a similar pattern with conduction reliability of stimulus APs (see Fig. 5), but the effect is more pronounced due to the fact that stimulus APs travel a shorter length $(l / 2)$ to the somatic end of the fiber. On the other hand, the conduction reliability of physiological APs decreases at each [stimulus frequency, physiological rate] pair for larger fiber diameters (see Fig. 4). We speculate that this is due to an increase in stimulus-physiological refractory period $\left(\Delta t^{\mathrm{II}}\right)$ and physiological-physiological refractory period $\left(\Delta t^{\mathrm{IV}}\right)$ (see Table 2 ).

The patterns seen in the standard deviations of physiological AP conduction map may be explained as follows. The physiological APs are generated from a Poisson process with rate $\lambda^{\text {phys }}$. Each physiological AP event has a probability of $p$ to not have an interaction as it travels up the fiber. This probability decreases as the stimulus frequency increases. Since the stimulus source is independent of the physiological source, the physiological APs that make it to the top of the fiber also generate a Poisson process with rate $p \lambda^{\text {phys }}$, which has a variance of $p \lambda^{\text {phys }}$. Thus, on the physiological AP conduction map, we should see (i) an increase in standard deviation with increasing $\lambda^{\text {phys }}$, (ii) decreasing standard deviation with increasing $f^{\text {stim }}$, and (iii) somewhat comparable standard deviation along diagonals. Indeed, this is what is observed in Fig. 4.

The patterns seen in the standard deviations in the stimulus AP conduction map are less straightforward. The only way a stimulus AP does not make it to the somatic end of the fiber is through loss of excitability in the middle of the fiber, which we compute statistics for in Fig. 6. Let the probability of loss of excitability in the middle of the fiber be $1-p$. Then, since the stimulus is deterministic, the process of stimulus APs making it to the somatic end of the fiber can be seen as a sequence of Bernoulli trials, with conduction probability being $p$. For values of $p$ away from 0.5 , the standard deviation $(\sqrt{p(1-p)})$ is small and increases as $p$ approaches 0.5 . From Fig. 6, $p$ increases towards 0.5 with increasing $f^{\text {stim }}$, more dramatically at low $\lambda^{\text {phys }}$ (moving from region 1 to 4 ), which increases the standard deviation as seen in the stimulus AP conduction map. Similarly, there is the least loss of excitability in the middle of the fiber for regions 1 and 2 (see Fig. 6), which means that $p$ is close to 0 and thus the standard deviation as seen in the stimulus AP conduction map is also small in these regions. Looking at the physiological AP map and stimulus AP map, the standard deviations have opposite trends, and appear to "cancel" each other out in the conduction map computed for all APs (Fig. 3).

Finally, a nerve fiber can be saturated if the frequency of stimulation is too high. The zero band in supplementary simulations (Fig. S1) is significant as the nerve fiber no longer responds to the stimulation and underlying physiological activity. We found that the large inward sodium current opposes the outward potassium current resulting in a net depolarization which makes the stimulus node unexcitable. The system remains in this state due to repetitive stimulation causing a prolonged blockage. 


\subsection{Interactions along the nerve fiber}

There have been experimental and computational efforts to demonstrate different interactions occuring on the nerve fiber (Iggo 1958; van den Honert and Mortimer 1981; Crago and Makowski 2014). In this study, we quantify a few of these interactions using a mechanistic model of myelinated nerve fiber. We find that the fraction of somatic APs from physiological and stimulus sources is constant (around 50\%) for $f^{\text {stim }} / \lambda^{\text {phys }}=1$. This is in agreement with results from (Crago and Makowski 2014). However, upon further examination of individual interaction counts (see Fig. 6c-f), we see that the pattern varies highly. Increasing the diameter increases $R_{\text {stim }}$ but decreases $R_{\text {phys }}$, which explains the increase in the fraction of end point APs due to the stimulus source.

Collision counts depend on the length of nerve fiber. As the length increases, the number of collisions increases since the conduction time for APs is longer. The low incidence of collisions in this simulation is due to the relatively short distance (and therefore short conduction time) between the physiological source and the stimulation site. Studies have shown this effect by varying the length of the nerve fiber or changing the location of stimulus (Crago and Makowski 2014). Interestingly, a $9 \mu \mathrm{m}$ nerve fiber has a higher collision count compared to $6 \mu \mathrm{m}$ fiber. We speculate that the relative difference in refractory periods (see Table 2) may cause this effect. For example, the physiological to stimulus refractory period decreases from $6 \mu \mathrm{m}$ fiber to 9 $\mu \mathrm{m}$ fiber (9.5 ms to $7.8 \mathrm{~ms}$ ), which increases the probability of collision for an upcoming physiological AP.

The physiological-physiological loss of excitability is very high in regions 2 and 5 (see Fig. 6d) on the conduction map. This is expected since the physiological rate is high. Since the physiological - physiological refractory period $\left(\Delta t^{\mathrm{IV}}\right)$ is increasing with respect to fiber diameter, we expect to see higher interactions for larger fibers. Similarly, the pattern is seen in stimulus - physiological loss of excitability as well. On the other hand, the physiological stimulus refractory period is decreasing with respect to fiber diameter. This leads to a decrease in the physiological stimulus interaction count. From Table 2, it is evident that physiological - stimulus refractory period is long and leads to the dominance of physiological - stimulus interactions. Refractory loss occurs with the least probability in Crago and Makowski (2014). This is contrary to our results because (Crago and Makowski 2014) uses a shorter refractory period in their study $(1.5 \mathrm{~ms})$ and they do not consider relative refractory period effects which occur in nerve fibers.

\subsection{Implications of the study}

Optimizing electrical stimulation to treat neurophysiological disorders has gained popularity in the field of neuromodulation. This problem can be approached on multiple fronts including electrode design, stimulation parameter optimization (e.g., frequency, amplitude), stimulation site, open versus closed system design etc. Multiple studies have looked into electrode design and stimulus location (Grill and Mortimer 1998; Tarler and Mortimer 2004; Crago and Makowski 2014). Few computational efforts have been made to optimize the stimulation parameters (McIntyre and Grill 1999; Foutz and McIntyre 2010). Even fewer computational efforts have been made in the design of closed-loop (Ehrens et al. 2015), partly due to the poor understanding of underlying interactions that must be captured in any model used for control design.

Most neurostimulation systems operate in an open-loop manner where the stimulation settings, like frequency, amplitude, and pattern, are preprogrammed. There is no feedback mechanism to dynamically update these parameters based on any physiological output. While the open-loop mechanism is simpler and provides short-term therapeutic benefits, it has suboptimal efficacy over longterm use. For example, open-loop spinal cord stimulation is used to treat chronic and acute pain. These systems may provide too much or too little strength of therapy since the stimulation parameters are not based on a patient's body position (Barolat et al. 1991; Olin et al. 1998). On the other hand, closed-loop neurostimulation systems can adjust their stimulation parameters in real-time and thereby provide effective and efficient therapies while also reducing the side effects of stimulation. Computational models used to design these systems can be fairly complex and thus affect real-time performance. This calls for reduced order models that can capture salient information as in higherorder mechanistic models but are computationally less complex.

In our previous study (Sadashivaiah et al. in press(a)), we built a reduced functional model based on the refractory periods, activation threshold and speed of conduction of a nerve fiber (similar to Crago and Makowski (2014)). Overall conduction reliability for the reduced model and mechanistic model (from this study) are very similar (see Fig. S4). The reduced model captures many conduction properties that the mechanistic model captures, but with significantly less time and less computation power. This is because the mechanistic model involves solving multidimensional differential equations at each fiber node to compute signal transmission. In contrast, the reduced model 
uses the activation threshold, the speed of conduction, and refractory periods to compute conduction statistics.

This study was motivated to model the pain pathways in the mammalian nociceptive network. Under normal conditions, both pain and sensory pathways mediate information in a relatively independent fashion. However, under pathological conditions, sensory pathways may pick up pain signals. Studies have shown that, under a sensitized condition (that is, injury), A-fiber mechano-receptors gain abnormal access to the nociceptive pathway and can induce pain (Baron 2009; Song et al. 2012). This suggests that both innocuous and noxious stimuli may travel along the same fibers up to the brain. An effective stimulation system must block the propagation of noxious stimuli while allowing the innocuous information to reach the brain. We can define a selective reliability parameter, $R_{\text {selective }}=R_{\text {innocuous }} \times$ ( $\left.1-R_{\text {noxious }}\right)$ to optimize this system using the conduction reliability parameters defined in this study. We analyze the selective reliability metric in a recent preliminary study (Sadashivaiah et al. in press(b)).

\subsection{Scope of study}

In our study, we constructed the underlying physiological activity as a Poisson process, with a mean firing rate of $1-50 \mathrm{~s}^{-1}\left(1-200 \mathrm{~s}^{-1}\right.$ for simulations in supplementary information). Although a Poisson process is a good representation of AP arrivals, the same mean firing rate can be obtained from infinitely-many different spike train patterns. Variable patterns (like bursts, etc.) may be more realistic characterizations of physiological response processes (Bruns et al. 2009). Similarly, stimulation with variable patterns may perform better in some applications. Refractory periods used in this study can vary depending upon the amplitude of stimulus and physiological inputs, biophysical properties of nerve fiber etc.

Phase resetting is an important type of interaction. This occurs in physiological AP generators when an antidromic AP reaches the physiological AP generation site before the next physiological AP would normally occur. With phase resetting, the voltage-dependent channels go to a state where another AP cycle cannot be generated. Hence, the next physiological AP will be generated after a regular wait time (interspike interval). Adding phase resetting to a homogeneous Poisson process does not change its firing properties. A homogeneous Poisson process assumes that the underlying instantaneous firing rate is constant over time, and the probability density function of the wait time or the interspike interval for a Poisson process is exponentially distributed and memoryless. A phase reset would just bring back the system to the same state of instantaneous firing. This assumption typically fails when the physiological input is drawn from other distributions.
This study was geared towards understanding the interactions between stimulus generated APs and underlying physiological activity generated APs. As a first step, we study interactions with a simple model of unbranched, multicompartmental nerve fiber. However, there are higher-order approximations of mammalian nerve fiber involving double cable architecture with discrete representations of internodal and paranodal sections (McIntyre et al. 2002). Using a different model of a nerve fiber will influence the reported results. More complex models and additional effects related to the fiber structure will be the subject of future studies. Stimulating electrodes can also take various shapes and configurations (Medtronic Neuromodulation 2007). There are numerous efforts to study the stimulation effects of these configurations (Yoshida and Horch 1993; Tyler and Durand 2002), and including different configurations is also future work.

Acknowledgments Work supported by NIH R01 AT009401 to S.V.S, Y.G., and W.S.A., and by NPRI postdoctoral fellowship awarded to P.S. We would like to thank Dr. Michael Caterina, Neurosurgery Pain Research Institute, The Johns Hopkins University School of Medicine for valuable and insightful discussions.

\section{Compliance with Ethical Standards}

Conflict of interests The authors declare that they have no conflict of interest.

\section{Appendix}

The fiber model and its parameters at $37^{\circ} \mathrm{C}$

\section{Fiber geometry}

axon diameter, $d=6-12 \mu \mathrm{m}$ (step size of $3 \mu \mathrm{m}$ )

ratio of axon to fiber diameter, $r_{\mathrm{dD}}=1$

fiber diameter, $D=d \times r_{\mathrm{dD}}$

ratio of $l_{\mathrm{i}}$ to fiber diameter, $r_{\mathrm{i}} \mathrm{D}=100$

internodal length, $l_{i}=D \times r_{\mathrm{li}_{\mathrm{i}} \mathrm{D}}$

fiber length, $L=10 \mathrm{~cm}$

nodal length, $l=2.5 \mu \mathrm{m}$

number of nodes, $n=\left\lceil 1+\frac{L}{l+l_{i}}\right\rceil$

Parameters

$$
\begin{aligned}
& \alpha_{\mathrm{mA}}=1.86, \alpha_{\mathrm{mB}}=65.6, \alpha_{\mathrm{mC}}=10.3 \\
& \alpha_{\mathrm{hA}}=0.0336, \alpha_{\mathrm{hB}}=-27, \alpha_{\mathrm{hC}}=11.0 \\
& \alpha_{\mathrm{nA}}=0.00789, \alpha_{\mathrm{nB}}=-9.2, \alpha_{\mathrm{nC}}=1.10 \\
& \alpha_{\mathrm{SA}}=0.00122, \alpha_{\mathrm{sB}}=71.5, \alpha_{\mathrm{SC}}=23.6 \\
& \beta_{\mathrm{mA}}=0.0860, \beta_{\mathrm{mB}}=61.3, \beta_{\mathrm{mC}}=9.16 \\
& \beta_{\mathrm{hA}}=2.30, \beta_{\mathrm{hB}}=55.2, \beta_{\mathrm{hC}}=13.4 \\
& \beta_{\mathrm{nA}}=0.0142, \beta_{\mathrm{nB}}=8, \beta_{\mathrm{nC}}=10.5 \\
& \beta_{\mathrm{sA}}=0.000739, \beta_{\mathrm{sB}}=3.9, \beta_{\mathrm{SC}}=21.8 \\
& \text { gating coefficients } \alpha_{* \mathrm{~A}}, \beta_{* \mathrm{~A}} \text { in } \mathrm{ms}^{-1}
\end{aligned}
$$


gating coefficients $\alpha_{* \mathrm{~B}}, \beta_{* \mathrm{~B}}, \alpha_{* \mathrm{C}}, \beta_{* \mathrm{C}}$ in $\mathrm{mV}$ sodium permeability, $P_{\mathrm{Na}}=7.04 \times 10^{-3} \mathrm{~cm} / \mathrm{s}$ potassium conductance (fast), $g_{\mathrm{Kf}}=0.015 \mathrm{~S} / \mathrm{cm}^{2}$ potassium conductance (slow), $g_{\mathrm{Kf}}=0.030 \mathrm{~S} / \mathrm{cm}^{2}$ leakage conductance, $g_{\mathrm{L}}=60 \times 10^{-3} \mathrm{~S} / \mathrm{cm}^{2}$ sodium concentration outside, $[\mathrm{Na}]_{\mathrm{o}}=154 \mathrm{mM}$ sodium concentration inside, $[\mathrm{Na}]_{\mathrm{i}}=35 \mathrm{mM}$ potassium concentration outside, $[\mathrm{K}]_{\mathrm{o}}=5.6 \mathrm{mM}$ potassium concentration inside, $[\mathrm{K}]_{\mathrm{i}}=155 \mathrm{mM}$ sodium equilibrium potential, $V_{\mathrm{Na}}=-84 \mathrm{mV}$ potassium equilibrium potential, $V_{\mathrm{K}}=+60 \mathrm{mV}$ resting membrane potential, $V_{\mathrm{r}}=-84 \mathrm{mV}$ Faraday constant, $F=96485 \mathrm{C} / \mathrm{mol}$ gas constant, $R=8.3144 \mathrm{~J} / \mathrm{Kmol}$ absolute temperature, $T=310.15 \mathrm{~K}$ membrane potential, $V \mathrm{mV}$

\section{Membrane currents}

sodium current, $I_{\mathrm{Na}} \mathrm{mA} / \mathrm{cm}^{2}$ potassium current (fast), $I_{\mathrm{Kf}} \mathrm{mA} / \mathrm{cm}^{2}$ potassium current (slow), $I_{\mathrm{Ks}} \mathrm{mA} / \mathrm{cm}^{2}$ leakage current, $I_{\mathrm{L}} \mathrm{mA} / \mathrm{cm}^{2}$

$I_{N a}=m^{3} h P_{\mathrm{Na}} \frac{V F^{2}}{R T} \frac{\left([\mathrm{Na}]_{\mathrm{o}}-[\mathrm{Naa}]_{i} e^{V F / R T}\right)}{\left(1-e^{V / R T}\right)}$

$I_{K f}=n^{4} g_{\mathrm{Kf}}\left(V-V_{\mathrm{K}}\right)$

$I_{K s}=s g_{\mathrm{Ks}}\left(V-V_{\mathrm{K}}\right)$

$I_{L}=g_{\mathrm{L}}\left(V-V_{\mathrm{L}}\right)$

\section{Simulation parameters}

simulation duration, $t_{\text {stop }}=30 \mathrm{~s}$

simulation step size, $t_{\text {step }}=0.001 \mathrm{~ms}$

simulation repeats, $n_{\text {rep }}=50$

\section{References}

Agarwal, R., \& Sarma, S.V. (2012). Performance limitations of relay neurons. PLoS computational biology, 8(8), e1002,626.

Bair, W., Koch, C., Newsome, W., Britten, K. (1994). Power spectrum analysis of bursting cells in area $\mathrm{mt}$ in the behaving monkey. Journal of Neuroscience, 14(5), 2870-2892.

Barolat, G., Zeme, S., Ketcik, B. (1991). Multifactorial analysis of epidural spinal cord stimulation. Stereotactic and functional neurosurgery, 56(2), 77-103.

Baron, R. (2009). Neuropathic pain: a clinical perspective. In Canning, B.J., \& Spina, D. (Eds.) Sensory Nerves (pp. 3-30). Springer.

Bruns, T.M., Bhadra, N., Gustafson, K.J. (2009). Bursting stimulation of proximal urethral afferents improves bladder pressures and voiding. Journal of Neural Engineering, 6(6), 1-8.

Crago, P.E., \& Makowski, N.S. (2014). Alteration of neural action potential patterns by axonal stimulation: the importance of stimulus location. Journal of Neural Engineering, 11(5), 1-9.

De Luca, C.J., LeFever, R.S., McCue, M.P., Xenakis, A.P. (1982). Behaviour of human motor units in different muscles during linearly varying contractions. The Journal of Physiology, 329(1), $113-128$.
Ehrens, D., Sritharan, D., Sarma, S.V. (2015). Closed-loop control of a fragile network: application to seizure-like dynamics of an epilepsy model. Frontiers in neuroscience, 9, 58.

Foutz, T.J., \& McIntyre, C.C. (2010). Evaluation of novel stimulus waveforms for deep brain stimulation. Journal of neural engineering, 7(6), 1-10.

Frankenhaeuser, B., \& Huxley, A.F. (1964). The action potential in the myelinated nerve fibre of Xenopus laevis as computed on the basis of voltage clamp data. The Journal of Physiology, 171(2), 302-315.

Grill, W.M., \& Mortimer, J.T. (1998). Stability of the input-output properties of chronically implanted multiple contact nerve cuff stimulating electrodes. IEEE Transactions on Rehabilitation Engineering, 6(4), 364-373.

Groves, D.A., \& Brown, V.J. (2005). Vagal nerve stimulation: a review of its applications and potential mechanisms that mediate its clinical effects. Neuroscience \& Biobehavioral Reviews, 29(3), 493-500.

Hines, M.L., \& Carnevale, N.T. (1997). The NEURON simulation environment. Neural Computation, 9(6), 1179-1209.

van den Honert, C., \& Mortimer, J.T. (1981). A technique for collision block of peripheral nerve: Frequency dependence. IEEE Transactions on Biomedical Engineering, 5(28), 379-382.

Hursh, J.B. (1939). Conduction velocity and diameter of nerve fibers. American Journal of Physiology, 127(1), 131-139.

Iggo, A. (1958). The electrophysiological identification of single nerve fibres, with particular reference to the slowest-conducting vagal afferent fibres in the cat. The Journal of Physiology, 142(1), $110-126$.

Jänig, W., Grossmann, L., Gorodetskaya, N. (2009). Mechano-and thermosensitivity of regenerating cutaneous afferent nerve fibers. Experimental Brain Research, 196(1), 101-114.

Kajander, K.C., \& Bennett, G.J. (1992). Onset of a painful peripheral neuropathy in rat: a partial and differential deafferentation and spontaneous discharge in a beta and a delta primary afferent neurons. Journal of Neurophysiology, 68(3), 734-744.

Katz, B. (1950). Action potentials from a sensory nerve ending. The Journal of Physiology, 111(3-4), 248.

Kralj, A.R., \& Bajd, T. (1989). Functional electrical stimulation: standing and walking after spinal cord injury. Boca Raton: CRC Press.

McIntyre, C.C., \& Grill, W.M. (1999). Model-based design of stimulus waveforms for selective microstimulation in the central nervous system. In Proceedings of the First Joint BMES/EMBS Conference, (Vol. 1 p. 384).

McIntyre, C.C., Richardson, A.G., Grill, W.M. (2002). Modeling the excitability of mammalian nerve fibers: influence of afterpotentials on the recovery cycle. Journal of Neurophysiology, 87(2), 995-1006.

McNeal, D.R. (1976). Analysis of a model for excitation of myelinated nerve. IEEE Transactions on Biomedical Engineering, 23(4), 329337.

Medtronic Neuromodulation (2007). Technical design summary: Model 39565 specify 5-6-5 surgical lead.

Mortimer, J.T., \& Bhadra, N. (2004). Peripheral nerve and muscle stimulation. In Neuroprosthetics: Theory and Practice, World Scientific (pp. 638-682).

Olin, J.C., Kidd, D.H., North, R.B. (1998). Postural changes in spinal cord stimulation perceptual thresholds. Neuromodulation: Technology at the Neural Interface, 1(4), 171-175.

Peckham, P.H., \& Kilgore, K.L. (2013). Challenges and opportunities in restoring function after paralysis. IEEE Transactions on Biomedical Engineering, 60(3), 602-609.

Pfurtscheller, G., Müller, G.R., Pfurtscheller, J., Gerner, H.J., Rupp, R. (2003). 'Thought'-control of functional electrical stimulation 
to restore hand grasp in a patient with tetraplegia. Neuroscience Letters, 351(1), 33-36.

Reilly, J.P. (1989). Peripheral nerve stimulation by induced electric currents: exposure to time-varying magnetic fields. Medical and Biological Engineering and Computing, 27(2), 101-110.

Sacré, P., Sarma, S.V., Guan, Y., Anderson, W.S. (2015). Electrical neurostimulation for chronic pain: on selective relay of sensory neural activities in myelinated nerve fibers. In Proceeding of the 37th Annual International Conference of the IEEE Engineering in Medicine and Biology Society (EMBC) (pp. 4705-4708).

Sadashivaiah, V. (2018). Modeling the interactions in a mammalian nerve fiber. https://github.com/vjysd/nerve-fiber-modeling.

Sadashivaiah, V., Sacré, P., Guan, Y., Anderson, W.S., Sarma, S.V. (2017). Modeling electrical stimulation of mammalian nerve fibers: a mechanistic versus probabilistic approach. In Proceeding of the 39th Annual International Conference of the IEEE Engineering in Medicine and Biology Society (EMBC) (pp. 3868-3871).

Sadashivaiah, V., Sacré, P., Guan, Y., Anderson, W.S., Sarma, S.V. (2018). Studying the interactions in a mammalian nerve fiber: a functional modeling approach. In Proceeding of the 40th Annual International Conference of the IEEE Engineering in Medicine and Biology Society (EMBC) (pp. 3525-3528). https://doi.org/10.1109/EMBC.2018.8512975, https://ieeexplore. ieee.org/document/8512975.

Sadashivaiah, V., Sacré, P., Guan, Y., Anderson, W.S., Sarma, S.V. (2018). Selective relay of afferent sensory-induced action potentials from peripheral nerve to brain and the effects of electrical stimulation. In Proceeding of the 40th Annual International Conference of the IEEE Engineering in Medicine and Biology Society (EMBC) (pp. 3606-3609). https://doi.org/ 10.1109/EMBC.2018.8513029, https://ieeexplore.ieee.org/document/ 8513029.

Schwarz, J.R., Reid, G., Bostock, H. (1995). Action potentials and membrane currents in the human node of ranvier. Pflü,gers Archiv, 430(2), 283-292.

Shealy, C.N., Mortimer, J.T., Reswick, J.B. (1967). Electrical inhibition of pain by stimulation of the dorsal columns: preliminary clinical report. Anesthesia \& Analgesia, 46(4), 489-491.
Shechter, R., Yang, F., Xu, Q., Cheong, Y.K., He, S.Q., Sdrulla, A., Carteret, A.F., Wacnik, P.W., Dong, X., Meyer, R.A., et al. (2013). Conventional and kilohertz-frequency spinal cord stimulation produces intensity-and frequency-dependent inhibition of mechanical hypersensitivity in a rat model of neuropathic pain. Anesthesiology, 119(2), 422-432.

Song, Y., Li, H.M., Xie, R.G., Yue, Z.F., Song, X.J., Hu, S.J., Xing, J.L. (2012). Evoked bursting in injured $\mathrm{A} \beta$ dorsal root ganglion neurons: a mechanism underlying tactile allodynia. Pain, 153(3), $657-665$

Stidd, D.A., Wuollet, A., Bowden, K., Price, T., Patwardhan, A., Barker, S., Weinand, M.E., Annabi, J., Annabi, E. (2012). Peripheral nerve stimulation for trigeminal neuropathic pain. Pain Physician, 15(1), 27-33.

Struijk, J.J., Holsheimer, J., Van Veen, B., Boom, H.B. (1991). Epidural spinal cord stimulation: calculation of field potentials with special reference to dorsal column nerve fibers. IEEE Transactions on Biomedical Engineering, 38(1), 104-110.

Tarler, M.D., \& Mortimer, J.T. (2004). Selective and independent activation of four motor fascicles using a four contact nerve-cuff electrode. IEEE Transactions on neural systems and rehabilitation engineering, 12(2), 251-257.

Troy, J., \& Robson, J. (1992). Steady discharges of $\mathrm{x}$ and $\mathrm{y}$ retinal ganglion cells of cat under photopic illuminance. Visual neuroscience, 9(6), 535-553.

Tyler, D.J., \& Durand, D.M. (2002). Functionally selective peripheral nerve stimulation with a flat interface nerve electrode. IEEE Transactions on Neural Systems and Rehabilitation Engineering, 10(4), 294-303.

Wall, P.D., \& Sweet, W.H. (1967). Temporary abolition of pain in man. Science, 155(3758), 108-109.

Wesselink, W.A., Holsheimer, J., Boom, H.B. (1999). A model of the electrical behaviour of myelinated sensory nerve fibres based on human data. Medical and Biological Engineering and Computing, $37(2), 228-235$.

Yoshida, K., \& Horch, K. (1993). Selective stimulation of peripheral nerve fibers using dual intrafascicular electrodes. IEEE transactions on biomedical engineering, 40(5), 492-494. 